\title{
Some determining factors of the alphanumeric category effect
}

\author{
KIM M. CARDOSI \\ Brown University, Providence, Rhode Island
}

\begin{abstract}
The alphanumeric category effect refers to the finding that letters and digits are identified more efficiently when presented among items from the opposite category (a between-category, BC, search) than among items from the same category (a within-category, WC, search). In Experiment 1, the category effect was demonstrated for some targets, but not for others. The reasons for the selectivity of the effect were clarified by Experiment 2, in which the magnitude of the effect was correlated with the physical similarities between targets and distractors. Experiment 3 showed that, in a BC search, the physical differences between letters and digits allowed the observer to be more accurate in locating a target than in identifying it. This was not the case in a WC search, in which the observer was more accurate in identifying a target than in locating it (Experiment 4). The conclusion of these experiments is that the alphanumeric category effect is dependent upon the physical aspects of the stimulus and not the perceptual categories.
\end{abstract}

Two of the most well-learned artifactual (i.e., nonnatural) categories are letters and numbers. These categories are well defined, even though the differences between them are difficult to define perceptually. The most distinguishing feature of these two categories is a functional one. In many visual search tasks it has been found that the ease with which a target can be detected depends, to a large extent, on the relationship between the categories of the target and the other items in the display. Many experiments have shown that it is easier to find a target among distractors from the opposite category (e.g., a letter among digits) than to find a target among distractors from the same category (e.g., a letter among other letters). This alphanumeric category effect has been found in both scanning tasks in which the subject searches for a target among a list of many items with unlimited viewing time (e.g., Brand, 1971; Ingling, 1972) and in the more commonly used visual search paradigm in which the number of distractors is small and varied and the display time is often limited (e.g., Gleitman \& Jonides, 1976, 1978; Jonides \& Gleitman, 1972, 1976).

Even when the existence of the alphanumeric category effect seemed indisputable, the mechanism behind this effect has remained controversial. Early explanations of the effect were based on the idea that category information (i.e., whether a character is a letter or digit) is accessed before the character's name. This explanation was supported by the finding that it is easier to categorize a tar-

\footnotetext{
This research was conducted at Brown University and was supported, in part, by Grant HDO5331 from the National Institute of Child Health and Human Development to Peter D. Eimas. I thank Peter D. Eimas for his guidance and critical comments throughout all stages of this work. I also thank Bryan E. Shepp and Kathryn T. Spoehr for their helpful comments and discussions. I am also grateful to the two anonymous reviewers for their comments. The author's present address is: U.S. Department of Transportation, Transportation Systems Center, DTS-45. Kendall Square, Cambridge, MA 02142.
}

get item as a letter or digit than it is to identify one target as, say, a Z or a 4 (e.g., Brand, 1971; Gleitman \& Jonides, 1976; Ingling, 1972). Duncan (1983) argued that when the effect is obtained with a single target, it is due to the physical similarities between letters and digits; on the average, items are physically more similar to items in the same category than to items in the other category. According to this view, a 4 would be easier to extract from a background of letters (i.e., in a between-category search) than from a background of digits (i.e., in a within-category search) because it is more physically distinct from individual letters than it is from other digits. In this view, categories per se have no causal relationship to the category effect.

In experiments in which the similarity between letters and numbers is manipulated, physical similarity has proven to be more powerful than categorical differences. Corcoran and Jackson (1977) had subjects search for a C, 6, A, or 4 among curved or angular distractors. Response times for finding a target among dissimilar distractors were significantly lower than those for finding a target among similar distractors, independently of whether it was a between-category or within-category search. Thus, feature similarity between targets and distractors had a significant effect on response time, whereas a categorical difference between them did not. Similarly, Krueger (1984) paired letter and digit targets for similarity, having subjects search for a $5, S, 6$, or G. Response time was shorter and accuracy was higher for finding a target among letter distractors than among digit distractors whether the target was a letter or a digit. Conversely, Egeth, Atkinson, Gilmore, and Marcus (1973) found lower reaction times for finding a target among digits than for finding one among letters whether the target was an A or a 4. None of these studies showed an overall category effect. Furthermore, whether it was easier to find a target among letters or among digits was idiosyncratic to the 
targets and distractors used in each experiment. One possibility is that the features of the targets and distractors were crucial in determining the ease of target detection. If feature similarity between targets and distractors can override the category effect, then it is possible that the effect is due solely to the physical differences between letters and numbers.

Experiments 1 and 2 investigated whether the locus of the category effect lay at the level of features or at the level of categories. The term "category information" will be used here to indicate knowledge that a given character is a letter or a digit. Since letters and numbers are very familiar and distinct categories, this information is considered to be easily, if not automatically, accessed after the character's name is accessed. These experiments went beyond demonstrating the presence or absence of the category effect, since they examined how the effect varied with different targets in the same background. Experiment 2 offers an explanation for this variability, since it correlated the magnitude of the difference between performance in between- and within-category searches with the magnitudes of the physical similarities between targets and distractors. Experiments 3 and 4 explored how the perceptual system uses this information on relative physical similarity to identify a target faster and more accurately in between-category search than in withincategory search.

\section{EXPERIMENT 1}

The idea that a between-category search is more efficient than a within-category search because of physical cues was discredited by the Jonides and Gleitman (1972) "Oh-zero" effect. Jonides and Gleitman had some subjects search for an $\mathrm{A}, \mathrm{Z}$, or $\mathrm{O}$ and other subjects search for a 2,4 , or $O$. The critical factor in this experiment was that the "oh" and the "zero" were physically identical. Nevertheless, the category effect was found with these ambiguous characters as well as with the unambiguous characters $A, Z, 2$, and 4 . On this basis, it was concluded that the necessary and sufficient condition for the category effect was a categorical difference between the target and its distractors.

Jonides and Gleitman's argument lost much of its force when Duncan (1983) failed to replicate the "oh-zero" effect using the same procedures. Duncan did, however, obtain the category effect with the unambiguous targets $A, Z, 2$, and 4 . From the failure to find any category effect on the sole basis of the category information given to the subject, Duncan concluded that the effect was due to the "average physical resemblance" among letters and numbers. However, he specified that this explanation held only for search for a single specified target. Duncan explained that, with more than one target, the category effect was due to the fact that in a between-category search the subject could use the well-learned letter-number distinction to differentiate targets from distractors. In a within-category search, however, the target and distractor sets are defined arbitrarily by the experimenter, and the subject must differentiate targets from distractors solely on the basis of the distinctions defined by the experimenter. Since this distinction is not as familiar or as well-practiced as the letter-number distinction, it is not as useful to the subject in the search task.

The present experiment evaluated the general validity of Duncan's ideas since it examined the role of feature information in the category effect. Subjects searched for letters or digits in both between-category (BC) and withincategory (WC) search tasks. Some of the targets were ambiguous in that they could be viewed as either letters or digits. This permitted a separation between the effects due to a categorical difference between targets and distractors and the effects of physical differences between letters and digits.

Most studies on the category effect use response time (RT) as the dependent measure; the present experiment also examined response accuracy in this four-alternative forced-choice search task. Although these two measures are generally assumed to be dependent on the same processes, Santee and Egeth (1982) have shown that they are differentially sensitive to early and late stages of processing under data-limited and resource-limited viewing conditions. One of the issues of interest in this experiment was the nature of the information that is used in the earliest stages of processing. To investigate this, the display duration on some trials was kept very brief. Under these conditions, error rates would be expected to be high and RTs might not be sensitive to factors that affect the earliest stages of processing. Therefore, accuracy was the dependent measure of interest when display duration was brief.

Very brief display durations were included in order to examine the differences in performance on a $\mathrm{BC}$ versus a WC search when the opportunity for information extraction was minimal. If the information responsible for the category effect is available at the earliest stages of processing, the effect should be apparent even with limited display exposure. If, however, the information takes time to derive, the effect should not be seen with very brief display durations and would become apparent only with longer durations. Both accuracy and RT were examined to measure the effect at longer display durations.

\section{Method}

Design. Each experimental session was divided into two parts. In one part, the targets appeared among distractors from the same category (a WC search), and in the other part, the targets appeared among distractors from the opposite category (a BC search). The order of the type of search was balanced across subjects so that half of the subjects performed the BC search first and half of them performed the WC search first. The experimental trials were blocked within display durations, and the order of target presentation within a block was random. The duration of the stimulus array was varied from 10 to $150 \mathrm{msec}$ at settings of $10,15,20,50$, and $150 \mathrm{msec}$. To counterbalance the effects of practice, the order of display du- 
rations was as stated above (10 to $150 \mathrm{msec}$ ) for half of the subjects and was reversed (150 to $10 \mathrm{msec}$ ) for the other half. The subjects received 48 trials at each display duration for a total of 240 trials for each search task.

Subjects. The subjects were 3 graduate and 9 undergraduate students at Brown University. All subjects reported normal or corrected-to-normal vision and were paid for their participation in a single 90 -min session.

Apparatus. Stimulus cards were presented in a mirror tachistoscope (Iconix Model 6137-4). The trial began with the presentation of a fixation point followed by the stimulus card. The luminance of the target field in the presence of a blank white card was $82 \mathrm{~cd} / \mathrm{m}^{2}$. After the target field disappeared, a blank field of the same luminance appeared and remained on until the next trial.

Stimulus materials. Each stimulus card contained one target and five distractors located on an imaginary circle with a diameter of $3.4^{\circ}$. The center of the circle corresponded with the predisplay fixation point. Targets and distractors consisted of uppercase letters and digits. For half of the subjects, the targets were I, E, S, and L. For the other subjects, the targets were 1, 3, 5, and 7. The letter distractors used were $\mathrm{C}, \mathrm{D}, \mathrm{H}, \mathrm{P}$, and $\mathrm{U}$; the digit distractors were $2,4,6,8$, and 9 .

The targets and distractors were Letraset 28-pt Futura Medium. These targets subtended $.44^{\circ}$ in height; targets other than I and 1 averaged $.31^{\circ}$ in width. (See Figure 1 for an illustration of the targets and distractors used.) The I in the typeface-a vertical linewas used as both the I and the 1 . The $\mathrm{L}$ was inverted and also used as the 7 in order to maximize the similarity between letter and digit targets.

Each target appeared at each of 12 locations on the imaginary circle corresponding to the 12 clock positions $(12: 30,1: 30, \ldots$, 11:30). One distractor was placed diagonally opposite the target, and the remaining distractors were placed randomly with the stipulation that no more than three characters could appear adjacent. In order to avoid the appearance of words or pronounceable nonwords in the display, a vowel was never placed between two consonants.

On half of the stimulus cards for each target set, the target appeared among distractors from the same category (a WC search); on the other half of the stimulus cards, the target appeared among distractors from the opposite category (a BC search).

Procedure. At the beginning of each half of the session, the subjects were shown both the targets and the distractors to be used. Twelve practice trials (three for each target) were conducted with a 200-msec display of the stimulus array. At the beginning of each trial, the experimenter said "go," at which point the predisplay fixation point appeared for $1 \mathrm{sec}$. The stimulus card immediately followed the offset of the fixation point. The subjects were instructed to look at the fixation point and to then identify which one of the four targets had been present in the array, guessing when necessary.

TARGETS:
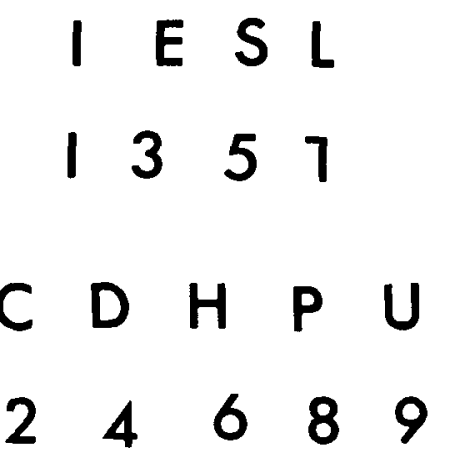

Figure 1. Targets and distractors used in Experiment 1 (Futura Medium).
Subjects searching for letter targets responded with a left-hand buttonpress for the $\mathrm{I}$ or the $\mathrm{E}$ and with a right-hand buttonpress for the S or the L. Subjects searching for digit targets pressed the left-hand button for the 1 or the 3 and the right-hand button for the 5 or the 7 . After depressing the appropriate response button, the subjects informed the experimenter as to which of the two targets (e.g., the I or the E) they had seen. The subjects were informed that their RTs were being monitored and were instructed to respond as quickly as possible without making mistakes. RTs from trials on which subjects accidentally pressed the wrong button (and informed the experimenter of this) were discarded, and those trials were repeated later in the session. (The incidence of this type of error was low, averaging approximately $5 \%$, and the RTs were noted to be similar to those for correct responses.) Trials on which the RTs were less than 200 msec or greater than $2 \mathrm{sec}$ were also disregarded and repeated later in the session (such occurrences were quite infrequent).

\section{Results and Discussion}

Response accuracies and RTs were recorded. In order to be comparable with other studies on the category effect, data with low error rates were needed for RT analysis. The mean RTs for each subject were computed from the block of trials at the lowest display duration on which the subject was at least $92 \%$ accurate. ${ }^{1}$ They were not computed from the same display duration for all subjects because the amount of practice that a subject received prior to any particular display duration was dependent upon the order of display durations presented, and this, in turn, affected accuracy at that duration. The means were taken from only one display duration (rather than all durations that met the criterion) so that they would all be based on the same number of trials.

The RT and accuracy data were both first analyzed for the effects of the order of display durations (150 to $10 \mathrm{msec}$ vs. 10 to $150 \mathrm{msec}$ ) and for the effects of the order of search type (BC or WC first). Neither factor had a significant effect on RT or response accuracy $[F(1,8) \leq 5.0, p>.05]$, nor did either factor interact with the effect of a BC or WC search (i.e., the category effect) $[F(1,8) \leq 2.2, p>.05]$. Since these factors showed no reliable main effect or interactions, they were not considered in the remaining analyses.

Since most experiments on the category effect use RT as the dependent measure, the RT data will be discussed first. Mean RTs from Experiment 1 for each target in each type of search are displayed in Table 1. RT data were analyzed in a three-way analysis of variance-category of targets (letters or digits) $\times$ (individual) targets $\times$ search type (BC or WC). Since different subjects searched for letter and digit targets, subjects were nested within targets, which were, in turn, nested within category of the targets. Whether the target was a letter or a digit had no effect $(F<1)$. However, there was a significant effect of targets within the category of the target $[F(6,42)=4.08$, $p<.01]$ as the RTs were lower for targets 1 , $\mathrm{I}$, and $\mathrm{L}$ than for the other targets in the same category.

Of more interest is the fact that RTs were significantly lower for a BC search than for a WC search $[F(1,14)=$ $9.25, p<.011$, indicating an overall category effect. 
Table 1

Mean Response Times (Milliseconds) for the Within-Category (WC) and Between-Category (BC) Search Tasks and the Differences Between Them for Experiment 1

\begin{tabular}{lrrrrrrrr}
\hline Search & \multicolumn{8}{c}{ Targets } \\
\cline { 2 - 9 } \multicolumn{1}{c}{ Task } & $\mathbf{1}$ & $\mathbf{3}$ & 5 & \multicolumn{1}{c}{7} & I & E & S & L \\
\hline WC & 612 & 751 & 745 & 728 & 702 & 781 & 784 & 710 \\
BC & 598 & 627 & 632 & 698 & 711 & 648 & 750 & 654 \\
WC-BC & 14 & 124 & 113 & 30 & -9 & 133 & 34 & 56 \\
\hline
\end{tabular}

Overall mean RTs were lower for a BC search than for a WC search for both digit targets (639 and 709, respectively) and letter targets (691 and 744, respectively). However, there was also a significant interaction between individual targets within categories and search type $[F(6,42)=4.82, p<.01]$. Thus, the effect of a BC versus WC search was not the same for each target. To determine which targets showed a significant category effect, the means for a BC and WC search for each target were compared using Fisher's least significant difference (L.S.D.) method (Winer, 1971). Within the letter target set, only the $\mathrm{E}$ and the $\mathrm{L}$ showed a significantly lower mean RT for a BC search than for a WC search [ $t(42)$ $=5.0, p<.01$ and $t(42)=2.1, p<.05$, respectively]. Neither the I nor the $S$ showed a reliable category effect $[t(42) \leq 1.3, p>.05]$. Within the digit targets, only the 3 and the 5 showed a significantly lower mean RT for a BC search than for a WC search $[t(42) \leq 4.3, p<.01]$. The category effect was not reliable for the 1 or the 7 $[t(42) \leq 1.1, p>.05]$. Thus, the overall category effect found was due to the targets $3,5, \mathrm{E}$, and $\mathrm{L}$, to varying degrees; the 1, 7, I and S showed no such reliable effect.

This relationship between individual targets and the category effect is also reflected in the accuracy data. Table 2 shows the mean percentage correct as a function of display duration for $\mathrm{BC}$ and $\mathrm{WC}$ searches and the corresponding $B C$ advantage (category effect). A repeatedmeasures analysis of variance of categories of targets $X$ (individual) targets nested within category of targets $x$ display duration $\times$ search type $\times$ subjects was performed. As found in the RT data, whether the target was a letter or a digit had no effect $(F<1)$ but the effect of targets within category was significant $[F(6,42)=5.5, p<.01]$; overall accuracy was lower for the E, S, and 7 than for the other targets in the same categories. The effect of display duration was also significant $[F(4,56)=52.6$, $p<.01]$, with accuracy increasing as display duration increased. Most important is the fact that a significant category effect was found, with accuracies being significantly higher for BC search than for WC search $[F(1,14)$ $=21.1, p<.01]$. There was also a significant interaction of target and search type $[F(6,42)=5.7, p<.01]$, indicating that, just as in the RT data, the effect of search type varied with the individual targets. No other interactions were significant.

Since there was not a significant interaction between display duration and category effect $[F(4,56)=1.6$, $p>.05]$, the mean accuracies for the $\mathrm{BC}$ search and the WC search were collapsed across display durations for comparisons within individual targets. These means are displayed in Table 3. Overall mean accuracies were higher for a BC search than for a WC search for both digit targets (.90 and .83 , respectively) and letter targets (.92 and .87 , respectively). Again, the means for a $\mathrm{BC}$ and $\mathrm{WC}$ search for each target were analyzed using the L.S.D. method. Although most of these differences between means were in the direction of a category effect, only the targets $E$ and 3 showed significantly higher response accuracies for a BC search than for a WC search $[t(42) \geq$ $2.05, p<.05]$. Thus, the only targets that showed a category effect in the RT data and not in the accuracy data were the $\mathrm{L}$ and the 5 . However, it should be noted that, although there was no reliable category effect with the target 5 , every subject showed a higher mean accuracy for a BC search (mean $=94, S D=5$ ) than for a WC search (mean $=89, S D=9$ ). This was the only case, however, in which there was a distinct trend in the data that did not result in a statistically significant category effect. With targets that showed no effect, usually only half of the subjects performed better in the BC search. It should also be noted that the fact that the magnitude of the category effect varied with different targets is as important as the fact that some targets showed the category effect and some did not.

These results show that the degree to which search for a target among distractors from a different category is more efficient than search for a target among distractors from the same category varies with the targets and distractors used. The data show that different targets from the same category can show a strong, weak, or no category effect. Because these targets share the same category information, the key to the category effect must lie in the physical features of letters and digits. Just as Duncan (1983) showed no "oh-zero" effect, this experiment showed no I-1 effect with an identical vertical line

Table 2

Mean Response Accuracies for the Within-Category (WC) and Between-Category (BC) Search Tasks and the Differences Between Them as a Function of Display Duration for Experiment 1

\begin{tabular}{lccccc}
\hline \multirow{2}{*}{$\begin{array}{l}\text { Search } \\
\text { Task }\end{array}$} & \multicolumn{5}{c}{ Display Duration } \\
\cline { 2 - 6 } & 10 & 15 & 20 & 50 & 150 \\
\hline BC & .73 & .90 & .96 & .98 & .99 \\
WC & .63 & .84 & .91 & .95 & .95 \\
BC-WC & .10 & .06 & .05 & .03 & .04 \\
\hline
\end{tabular}

Table 3

Mean Response Accuracies for the Within-Category (WC) and Between-Category (BC) Search Tasks and the Differences Between Them for Experiment 1

\begin{tabular}{lllllllll}
\hline Search & \multicolumn{7}{c}{ Targets } \\
\cline { 2 - 9 } Task & 1 & 3 & 5 & 7 & I & E & S & L \\
\hline BC & .94 & .94 & .94 & .80 & .89 & .95 & .90 & .93 \\
WC & .92 & .82 & .85 & .75 & .93 & .81 & .84 & .91 \\
BC-WC & .02 & .12 & .09 & .05 & -.04 & .14 & .06 & .02 \\
\hline
\end{tabular}


being used for both targets. In the present experiment, however, the lack of a category effect was also shown with two unambiguous targets. The $S$ and 7 failed to show a category effect in either accuracy or RT. These absences of a category effect support the idea that the basis for the effect lies in physical differences between letters and digits. It is possible that the $S$ (the only curved letter target) and the 7 were too physically similar to their oppositecategory distractors to show any advantage for a BC search.

The fact that no interaction between display duration and search type was found suggests that the category effect was unaffected by the various limits imposed on the information available in the display. However, the effect was actually slightly stronger when the opportunity for information extraction was severely limited than when the viewing conditions were optimal. This indicates that whatever processes are responsible for producing the category effect operate in the early stages of the search. If it is true that category information comes in very early and produces the category effect, then the failure to find the effect with all targets cannot be explained. It seems more likely that physical feature information is responsible for the effect.

In most search tasks, the physical differences between a target and its distractors are crucial in determining the difficulty of the search (e.g., Neisser, 1964; Rabbitt, 1967). Similarly, the category effect may also be a function of the physical similarity between targets and distractors. When a letter or digit is more similar to the characters in its own category than to the characters in the opposite category, then it may be easier to detect in a BC search than in a WC search and no category effect should be found. This hypothesis was tested in Experiment 2, with the relationship between the category effect and the physical similarity between a target and its distractors being explored.

\section{EXPERIMENT 2}

Experiment 2 investigated the apparent dependency of the category effect on distinctive physical differences between letters and digits. The only difference between this experiment and Experiment 1 was the typeface usedliquid crystal display (L.C.D.) as opposed to Futura Medium. In the L.C.D. typeface, each letter and digit is based on a seven-segment display (see illustration in Figure 2). This type of display has a number of advantages. First, letters and digits are all angular and all have the same height and (with the exception of the I and 1) the same width. In effect, they are all physically similar. Second, this similarity can be easily quantified by considering the number of line segments that two characters would have in common if they were superimposed. The similarity between a target and a set of distractors can then be quantified by summing the number of elements shared by a target and each of its distractors. In this way, the similarity between any given target and distractor set can
TARGETS:

\section{$1 E 5 L$}

1357

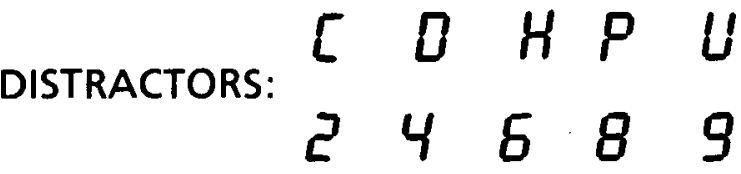

Figure 2. Targets and distractors used in Experiment 2 (L.C.D.).

be compared. In this experiment, to account for differences in performance on $\mathrm{BC}$ and $\mathrm{WC}$ search, the similarity of a target to its same- and different-category distractors was examined. The third advantage of this typeface is that it is very familiar to subjects as "digital," L.E.D. (light emitting diode) and/or L.C.D. (liquid crystal display).

In this experiment, the typical features of letters and digits that were present in Experiment 1 were not available, but the category information remained intact. If the category effect depends on the prototypical physical features of letters and digits, then no category effect would be expected with the targets that showed the category effect in Experiment 1. Furthermore, if it is true that category information cannot alter the effects of physical similarity between targets and distractors, then performance on the search tasks should be correlated with the similarity index and be independent of the type of search.

\section{Method}

Design. The design of Experiment 2 was the same as that of Experiment 1.

Subjects. The subjects were 12 undergraduate students at Brown University. All subjects reported normal or corrected-to-normal vision and were paid for their participation in the single 90-min session.

Apparatus. The apparatus used was the same as that described for Experiment 1.

Stimulus materials. The stimulus materials used were the same as those described for Experiment 1 with one exception. The typeface used was 24-pt Letraset L.C.D. (see Figure 2). These items subtended $.44^{\circ}$ in height and, with the exception of the $I / 1$, subtended $.25^{\circ}$ in width. The $S$ in the typeface was also used as the 5 , and the 3 was reversed and also used as the $E$. These stimuli were used to maximize the similarity between letter and digit targets. The only difference between the stimuli used in Experiment 1 and those used in Experiment 2 was the typeface used. For each stimulus card used in Experiment 1, there was a replica with the L.C.D. typeface in Experiment 2; the targets, distractors, and their positions were identical in both experiments.

Procedure. The procedure used in Experiment 2 was identical to that described for Experiment 1.

\section{Results and Discussion}

The data from Experiment 2 were analyzed in the same fashion as the data from Experiment 1. Again, the RT 
and accuracy data were both first analyzed for the effects of the order of display duration and for the effects of the order of search type. Neither factor had a significant effect on RT or response accuracy $[F(1,8) \leq 2.3, p>.05]$ and neither factor interacted with the category effect $[F(1,8) \leq 4.8, p>.05]$. Since these factors showed no reliable main effects or interactions, they were not considered in the remaining analyses.

Mean RTs are displayed in Table 4. Overall mean RTs were lower for a BC search than for a WC search only for the digit targets ( 644 and 725 , respectively). With letter targets, mean RTs were higher for a BC search than for a WC search (730 and 682, respectively). An analysis of variance showed a significant effect of targets within category of target $[F(6,42)=16.0, p<.01]$ as the mean RTs were lower for the targets I, L, 1, and 7 than for the other targets within their respective categories. As in Experiment 1, whether the target was a letter or a digit had no effect on RT $(F<1)$. More importantly, the effect of search type (BC vs. WC) was not significant $(F<1)$, indicating no overall category effect. Yet, just as in Experiment 1, there was a significant target within category of target $\times$ search type interaction $[F(6,42)=$ $69.4, p<.01]$. Again, the differences found between a $\mathrm{BC}$ and a WC search depended on the target. To determine which targets showed the category effect, differences between means for a BC and WC search were analyzed for each target using the L.S.D. approach. For the targets 3 and 5 , the mean RTs for a BC search were significantly lower than the mean RTs for a WC search [t(42) $\geq 3.3, p<.01]$. This difference is one that could be referred to as a category effect. No such effect was found for the targets $1,7, \mathrm{E}$, or $\mathrm{L}[t(42)<1.5]$. In fact, for the targets 1 and $E$, the mean RT for a BC search was actually higher than that for a WC search. This was also true for the target $S$, but in this case, the difference was significant; the mean RT was significantly higher for a $\mathrm{BC}$ search than for a WC search $[t(42)=-6.9, p<.01]$. This difference in RT is in the direction opposite to that of the category effect. Thus, whether RTs were lower in a BC or a WC search depended exclusively on the target.

The same trends are found in the accuracy data. As shown in the RT data, a BC search was more efficient than a WC search only for digit targets. For digit targets, the overall mean accuracies for a BC search and a WC search were .89 and .85 , respectively; for letter targets, the mean accuracies were .83 and .86 , respectively. Table 5 shows mean response accuracy as a function of dis-

Table 4

Mean Response Times (Milliseconds) for the Within-Category (WC) and Between-Category (BC) Search Tasks and the Differences Between Them for Experiment 2

\begin{tabular}{lrrrrrrrr}
\hline $\begin{array}{c}\text { Search } \\
\text { Task }\end{array}$ & \multicolumn{1}{c}{ 1 } & 3 & 5 & \multicolumn{1}{c}{ Targets } & \multicolumn{1}{c}{ I } & \multicolumn{1}{c}{ E } & \multicolumn{1}{c}{ S } & L \\
\hline WC & 573 & 784 & 904 & 640 & 628 & 713 & 736 & 653 \\
BC & 604 & 668 & 722 & 584 & 591 & 764 & 976 & 590 \\
WC-BC & -31 & 116 & 182 & 56 & 37 & -51 & -240 & 63 \\
\hline
\end{tabular}

Table 5

Mean Response Accuracies for the Within-Category (WC) and Between-Category (BC) Search Tasks and the Differences Between Them as a Function of Display Duration (Milliseconds) for Experiment 2

\begin{tabular}{|c|c|c|c|c|c|}
\hline \multirow{2}{*}{$\begin{array}{c}\text { Search } \\
\text { Task }\end{array}$} & \multicolumn{5}{|c|}{ Display Duration } \\
\hline & 10 & 15 & 20 & 50 & 150 \\
\hline $\mathrm{BC}$ & .58 & .86 & .94 & .96 & .96 \\
\hline WC & .61 & .84 & .92 & .94 & .96 \\
\hline$B C-W C$ & -.03 & .02 & .02 & .02 & .00 \\
\hline
\end{tabular}

Table 6

Mean Response Accuracies for the Within-Category (WC) and Between-Category (BC) Search Tasks and the Differences Between Them for Experiment 2

\begin{tabular}{lcccccccc}
\hline \multirow{2}{*}{$\begin{array}{l}\text { Search } \\
\text { Task }\end{array}$} & \multicolumn{1}{c}{ Targets } \\
\cline { 2 - 10 } BC & 3 & 5 & 7 & I & \multicolumn{1}{c}{ E } & \multicolumn{1}{c}{ S } & L \\
WC & .93 & .90 & .87 & .87 & .90 & .80 & .69 & .93 \\
BC-WC & -.94 & .82 & .77 & .86 & .89 & .82 & .82 & .93 \\
\hline
\end{tabular}

play duration for both a BC and WC search and the resulting BC advantage. There was an effect of display duration, as accuracies increased with display duration $[F(4,56)=69.4, p<.01]$. There was also a significant effect of target within category of target $[F(6,42)=12.5$, $p<.01]$, as overall accuracies were highest for 1 , I and $\mathrm{L}$ and lowest for 5 and $\mathrm{S}$. This effect of target was most pronounced at the lower display durations, yielding a target $\times$ display duration interaction $[F(24,168)=5.2$, $p<.01]$. As with the RT data, there was no overall effect of category of target (letter or digit) or search type ( $F<1$ in both cases), but the target within category of target $\times$ search type interaction was significant $[F(6,42)$ $=5.1, p<.01]$. No other interaction was significant.

As in Experiment 1, performance on a BC search as compared with a WC search differed with individual targets and these differences in the accuracy data closely parallel those found in the RT data. Mean accuracies (collapsed across display durations) for each target are displayed in Table 6. Mean accuracy for a BC search was significantly higher than accuracy for a WC search for both the 3 and the $5[t(42)=2.8, p<.01$, and $t(42)$ $=2.3, p<.05$, respectively]. The opposite was true for the target $S$, since mean accuracy was significantly lower for a BC search than for a WC search $[t(42)=-3.0$, $p<.01]$. Just as in the RT data, no significant effect was found with the I, E, L, 1 , or 7 ( $t<$ in all cases).

The results of Experiment 2 showed that the efficiency of a BC or a WC search depended on the physical differences between the target and its distractors. Although a strong category effect was found with the targets 3 and 5 , no such effect was found with the $1,7, I, E$, or L. Furthermore, with the targets $1, \mathrm{E}$, and $\mathrm{S}$, search was more efficient in a WC search than in a BC search. This strongly suggests that the physical features of the targets and distractors are crucial in the demonstration of a category effect. If category information was sufficient to 
produce the category effect, then a category effect should have been found with all of the targets. The strongest indictment against the sufficiency of category information alone comes from the results obtained with the targets $S$ and 5. Although a strong category effect was found with the target 5 , no such effect was found with the physically identical S. In fact, the advantage for the BC search for the 5 was of the same magnitude as the advantage for the WC search for the $S$. Since the 5 and the $S$ were identical in this typeface, it seems as though the category information contained in the $S$ could not abate the detrimental effects of the physical features of the digit distractors. Thus, performance on the search for the 5/S target was the same for the two groups of subjects. The target was significantly easier to recognize among letters than among digits, whether the target was called a 5 or an $S$.

It could be argued that the $5 / \mathrm{S}$ character was, in some way, better as a 5 than as an S. This is unlikely, however, since the ambiguous figure was actually the L.C.D. S. Still, the figure may have been more familiar to the subjects as a 5 than as an $S$, since this typeface is most frequently encountered in numerical displays (e.g., a digital clock). Similarly, it could be argued that no overall category effect was found in Experiment 2 because the L.C.D. typeface was too unfamiliar or somehow made the task too difficult. There are three sources of evidence that contradict these arguments. First, a preliminary experiment showed that the targets (when presented alone) were no more discriminable in the Futura Medium typeface than in the L.C.D. typeface. ${ }^{2}$ Second, overall mean accuracies and RTs for Experiments 1 and 2 were strikingly similar. ${ }^{3}$ Lastly, if familiarity of the target was crucial to a demonstration of the category effect, then the effect should have been observed with the prototypical $S$ in Experiment 1 .

The most likely explanation for the results of both experiments is that the physical similarity between the target and distractors determined performance on the search task. Table 7 shows each L.C.D. target with the total number of line segments in common between the target

Table 7

The Differences Between the Total Number of Line Segments Common to the Target and Each of its Distractors in the Between-Category (BC) and Within-Category (WC) Search Tasks, and the Corresponding Differences in Response Time (RT) and Accuracy Number of Common Line Segments

\begin{tabular}{crrrrrr} 
Target & BC & WC & Difference & & RT & Accuracy \\
\hline 1 & 10 & 9 & -1 & & -31 & -.01 \\
3 & 15 & 21 & 6 & 116 & .08 \\
5 & 16 & 21 & 5 & 182 & .10 \\
7 & 10 & 12 & 2 & 56 & .01 \\
I & 9 & 10 & 1 & 37 & .01 \\
E & 18 & 20 & -2 & -51 & -.02 \\
S & 21 & 16 & -5 & -240 & -.13 \\
L & 11 & 13 & 2 & 63 & .00 \\
\hline
\end{tabular}

and its two sets of distractors (BC and WC), the difference between these two sums (WC $-B C$ ), and the net differences in mean RTs and accuracies. The difference between the total number of segments in common will be referred to as the WC similarity index. The higher the index, the greater the similarity between the target and its WC distractors relative to its $\mathrm{BC}$ distractors. The direction of this difference is also very important. If the WC index is positive, then the target has more elements in common with the characters of its own category than with those of the opposite category. According to a similarity hypothesis, this should result in differences in performance between a WC and a BC search that would be referred to as a category effect. If, however, the WC index is negative, then the target has more elements in common with the distractors from the opposite category than it has with the distractors from the same category. This should result in RT and accuracy differences that are in the direction opposite to that of the category effect (i.e., a within-category advantage). This is, in fact, what was observed in Experiment 2. The WC similarity index is positively correlated with the mean RT differences $(r=.95)$ and the mean accuracy differences $(r=.95)$. Therefore, the results of Experiment 2 can be explained (with $90 \%$ of the variance accounted for) in terms of similarity between targets and distractors, without any reference to category information.

The results of Experiments 1 and 2 strongly suggest that the physical differences between letters and digits are responsible for the category effect. Not only is category information alone insufficient to produce the effect, but it also cannot nullify the detrimental effects of similarity between a target and its opposite-category distractors. To be certain that physical similarity was the determining factor in other experiments that demonstrate a category effect (or the lack of one), a point-by-point computer analysis of the degree of overlap between each target and each of its distractors could be done on the stimuli. However, that task is beyond the scope of this paper. Furthermore, while most of the studies that demonstrate the category effect specify a single target before each trial, the present experiments had subjects search for four targets simultaneously. In the present experiments, the categorically different targets could not be specified in terms of a given set of features as is possible with search for a single target. Nevertheless, it was the physical differences between letters and digits that were found to be responsible for the category effect. Thus, Duncan's distinction between the source of the category effect with one target and with more than one target is not necessary; with more than one target, as with only one target, it is still the "uncontrolled variation in the physical resemblance between targets and nontargets" (Duncan, 1983, p. 231) that is responsible for the category effect and not the well-known letternumber classification. The only difference is that with more than one target, the physical similarity between the 
targets will affect performance as will the similarity between the targets and distractors.

Now that the source of the category effect has been identified, more detailed hypotheses about the precise mechanisms of this effect can be explored.

\section{EXPERIMENT 3}

The results of the first two experiments showed that the key to the alphanumeric category effect lay in the physical differences between letters and numbers. How this information is used by the processing system to extract a categorically different item faster and more accurately than a target from the same category as its distractors was explored in Experiment 3. Even though the nature of the category effect is physical rather than categorical, the term "category effect" will continue to be employed as a matter of convenience and convention. Furthermore, it will be assumed that, generally, items in the same alphanumeric category will be more physically similar to each other than to items in the opposite category.

Jonides and Gleitman (1972, 1976; Gleitman \& Jonides, 1976) suggested that the decision as to whether an item is a digit or letter requires less detailed feature extraction than does the identification of that item. In their BC search task, the subject needed only to decide whether a categorically different item was, or was not, present. The results of Experiments 1 and 2 suggest that this decision could have been possible on the basis of physical dissimilarity. That is, the subjects may have actually decided whether or not a physically distinct, rather than a categorically distinct, item was present. In a WC search, however, each item in the display needed to be scrutinized in order to determine whether or not it was the target. This may be true only because the items within a category are very similar. The fact that finding a target in a BC search requires less detailed processing than finding a target in a WC search has been demonstrated by Gleitman and Jonides (1976). They found that subjects in a WC search remembered the distractors much better than did subjects in a BC search. In addition, Gleitman and Jonides showed that subjects who were practiced on a BC search tended to respond to any member of the target category as a target, even though it was not a member of the target set. (This is referred to as the "catch trial" effect.) If the subjects had fully processed the target before responding to it, they would not have made these false detections. Again, this result can be explained on the basis of physical dissimilarity. Subjects could have been responding to any physically distinct item before realizing that this criterion for identifying a target was too lax.

These two separate lines of evidence indicate that the processing of targets and distractors in a $\mathrm{BC}$ search differs from that in a WC search. In a WC search, each item must be fully processed, that is, identified, in order to decide whether it is a target or a distractor. In a BC search, a partial processing of the elements in the display may be sufficient to extract the categorically different item, since a target in a BC search will be more physically distinct than a target in a WC search. This initial partial processing of feature information is said to enable the subject to focus attention upon a particular location in the array in a BC search. In their view, the categorical difference between a target and its distractors "spatially tags" the target for further processing (Jonides \& Gleitman, 1976).

In the present view, it is the distinctive features between letters and digits found to be responsible for the category effect that allow the subject to focus attention on the target for identification. In this view, a "present/absent" judgment, such as that required in many of the experiments on the category effect, could be made on the basis of the tag alone. That is, the information that is believed to result in the spatial tag, that is, that there is a physically distinct item, is sufficient for a subject to be able to decide that a categorically different item is present. If the items are physically homogeneous, then a response of "absent" would be appropriate. This would not be the case, however, if the subject was searching for more than one target simultaneously (e.g., for " any digit"). In this case, the tag could only locate the target for further processing and not, in itself, serve as the basis for a response. The usefulness of such a tag in a search for multiple targets was examined in Experiment 3.

It has been suggested here that the relatively quick accessing of a target's location may be based on physical differences between a target and the distractors. This process facilitates the identification of different category items by attracting attention to, or focusing attention upon, the target for further processing. It is this location process, made possible by the physical differences between letters and digits, that is apparently responsible for the superior performance found in BC search.

If the above hypotheses are correct, then RT should be shorter and accuracy should be higher for the localization of a BC target than for its identification (see Jonides \& Gleitman, 1976). However, there is a problem with parcelling out RT due to locating the target and RT due to translating this location information into the appropriate response. There is no such problem with naming a target, since these responses are well learned and probably automatic. An alternative approach to trying to separate the two processes of target localization and target identification by measuring RT to each response is to monitor the accuracy of these two responses under display conditions that allow for minimal information extraction. When information available from a display is limited, the ability to extract location information relative to the ability to extract identity information should indicate which type of information is first utilized by the processing system. If a categorical (and physical) difference between a target and its distractors can indicate a target's location more efficiently than it can indicate a target's identity, then subjects should be more accurate at locating a target in a $\mathrm{BC}$ search than at identifying the same target. 
Experiment 3 was conducted to investigate the validity of these claims. In this experiment, subjects were asked to find an unspecified digit or letter (i.e., search for any digit or any letter) in a BC search. (Note that information as to the physical configurations of the targets is not as useful in this task as it is in tasks that specify a single target before each trial.) Subjects reported both the target's identity and its location as display duration was manipulated. According to the location hypothesis, a BC search should result in higher response accuracies for reporting the target's location than for reporting the target's identity. This should be most evident at brief display durations, in which the opportunity for information extraction is minimal. If it is, indeed, easier to extract location information than identity information, then subjects should be more accurate at reporting the target's location than its identity. This difference in accuracies should be most apparent when viewing conditions are severely limited so that it is difficult or impossible to determine both the location and the identity of the target.

\section{Method}

Design. The subjects were informed as to the target set and distractors and were told to search for any one of the target items, that is, to search for any digit or any letter. The subjects reported both the identity (name) and the location of the target shown and were told to guess when necessary. Location responses were given using eight compass directions (N, NE, E, .., NW). A sheet displaying these compass directions and the target set was placed in front of a subject, and the subject was encouraged to refer to it whenever necessary.

The subjects were divided into two groups; one searched for letters and the other searched for digits. Half of the observers in each of the two groups were instructed to respond with the identification response first and the location response second; for the other half, the order of responses was reversed. The order of stimuli was random and different for each subject. Response accuracies for both the identity and location responses were recorded.

Display durations were manipulated systematically from 10 to 200 msec. The six durations used were $10,15,25,50,100$, and $200 \mathrm{msec}$. All subjects received 64 blocked trials (the eight targets were shown at each of the eight locations) at each of the six display durations. For half of the subjects the order of display durations was as stated above (10 to 200 ); for the other half, the order was reversed $(200$ to 10$)$.

Subjects. The subjects were 2 graduate and 22 undergraduate students at Brown University. All subjects reported normal or corrected-to-normal vision and were paid for their participation in a single 1-h session.

Apparatus. The apparatus used was the same as that described for Experiment 1.

Stimulus materials. Each stimulus card contained one target and seven opposite-category distractors located on an imaginary circle with a diameter of $3.1^{\circ}$. The center of this circle of characters corresponded with the preexposure fixation point. Targets and distractors consisted of Arttec 18-pt Futura Demibold uppercase letters and digits. The letters averaged $.19^{\circ}$ in width, and the digits averaged $.15^{\circ}$ in width. Both letters and digits subtended $.25^{\circ}$ in height. The target set for half of the subjects consisted of the digits 1,2 , $3,4,5,6,7$, and 8 ; the targets for the other subjects were the letters $C, D, G, H, K, R, S$, and V. (These letters and digits are displayed in Figure 3.) These sets were chosen in an attempt to approximately match letter and digit targets for curvanure and linearity. In each case, all of the nontarget items were from the opposite category so that subjects searched for letter targets among digit dis-
TARGETS:

12345678

\section{DISTRACTORS: C D G H K R S V}

Figure 3. Targets and distractors used in Experiment 3.

tractors or for digit targets among letter distractors. The target set for one group was the distractor set for the other group. Each of the targets was shown equally often in each of the eight possible positions on the imaginary circle. Distractors were located randomly with the following restrictions: each target appeared adjacent to each distractor at least twice and, for each target, any given distractor could appear in the same position no more than three times. These steps were taken to prevent any distractor or set of distractors from becoming a cue to the target's location or identity.

Procedure. At the beginning of each session, the subjects were shown both the targets and the distractors. At the beginning of each trial, the experimenter said "go," at which point the predisplay fixation point appeared for $1 \mathrm{sec}$. The subjects were instructed to look at the fixation point and were told that the stimulus array would follow it immediately.

The subjects received 24 practice trials (three of each of the eight targets) with a display duration of $200 \mathrm{msec}$. If the responses on the practice trials were not $100 \%$ correct, more practice was given at an appropriately higher display duration. Practice was then given at intermediate durations, if necessary, until the criterion of eight consecutive correct responses with the $200-\mathrm{msec}$ display duration was met.

\section{Results and Discussion}

Response accuracies were corrected for guessing before analysis. ${ }^{4}$ Before the data were analyzed for differences in accuracies between location and identity responses, differences due to the order of display durations (i.e., increasing from 10 to 200 or decreasing from 200 to 10 ) and to the order of the identity (ID) and location (LOC) responses were assessed. For this purpose, a four-factor repeated-measures analysis of variancedisplay duration $x$ order of display durations $\times$ response order $\times$ target type (letters or digits)-was performed. The effects due to the order of display durations used and to response order were not reliable ( $F<1$ in each case). Furthermore, these factors did not interact significantly with the effect of ID versus LOC responses. They were therefore excluded from further consideration.

The differences between mean accuracies for ID and LOC responses as a function of display durations are depicted in Table 8 . The differences between them (LOC-ID) indicate the advantage of the location

Table 8

Mean Response Accuracies for Location (LOC) and Identification (ID) Responses and the Differences Between Them as a Function of Display Duration for Experiment 3

\begin{tabular}{|c|c|c|c|c|c|c|}
\hline \multirow{2}{*}{$\begin{array}{c}\text { Type of } \\
\text { Response } \\
\end{array}$} & \multicolumn{6}{|c|}{ Display Duration } \\
\hline & 10 & 15 & 25 & 50 & 100 & 200 \\
\hline LOC & .30 & .55 & .70 & $\begin{array}{r}.78 \\
78\end{array}$ & $\begin{array}{r}.81 \\
81\end{array}$ & .82 \\
\hline ID & .23 & .51 & .68 & .78 & .81 & \\
\hline LOC-ID & .07 & .04 & .02 & .00 & .00 & -.01 \\
\hline
\end{tabular}


responses over the identification responses. The mean accuracies are indeed slightly higher for location responses than for identity responses, until the two functions asymptote at the higher display durations. A three-factor repeated-measures analysis of variance-target type $\times$ response type (ID vs. LOC) $\times$ display duration was performed on the data. The subjects were significantly more accurate at reporting the target's location than at reporting the target's identity $[F(1,22)=8.13, p<.01]$. The magnitude of this difference was a function of display duration and was most apparent at the lowest display durations, and thus the significant response type by duration interaction $[F(5,110)=5.13, p<.01] .{ }^{5}$ In fact, Fisher's L.S.D. test showed that this difference was significant only with a $10-\mathrm{msec}$ display duration $[t(110)=4.1$, $p<.01]$ and with a 15-msec display duration $[t(110)=$ $2.4, p<.05]$. With a display duration of $50 \mathrm{msec}$ and higher, the two functions were virtually the same. This shows that the process that locates, or "tags," a BC target for identification occurs in the early stages of processing. When there is ample time to process the display, there is no advantage, at least in response accuracy, for the LOC over the ID responses.

Significantly higher response accuracies were also found with letters as targets than with digits as targets $[F(1,22)=14.15, p<.01]$. The overall mean accuracy for letter targets was .74, whereas that for digit targets was .56. This difference was most apparent at the lower display durations, resulting in the significant target type $\times$ duration interaction $[F(5,110)=3.35, p<.01]$. Lastly, there was a significant effect of display duration, as accuracy increased with display duration $[F(5,110)=$ $155.2, p<.01$ ]. No other interaction was found to be significant $(F<1$ in each case).

The most likely explanation for the higher accuracies for letters as targets than for digits as targets is that letter targets were more discriminable from their digit distractors than digit targets were from their letter distractors. On average, the letters used were wider than the digits. This would allow a digit target to stand out among letter distractors (as it is more narrow) and a letter target to stand out among digits (as it is wider). However, the most narrow characters were the $1 / \mathrm{I}$ and the letter $S$. The $S$ subtended $.13^{\circ}$, whereas every other letter except I subtended at least $.19^{\circ}$. The digits, on the other hand, averaged $.15^{\circ}$ in width. This had important consequences for search accuracy, as did the striking similarity between the $S$ and the 5 . It was found that the higher error rate for digit targets than for letter targets could be attributed to the fact that the S was a particularly efficient distractor. ${ }^{6}$

The width and shape of the $S$ made it more similar to the digits than to the other letters. This made the $S$ a very effective distractor for all of the digit targets, as it attracted attention toward itself and away from the target. This greater similarity between the $S$ and the digits than between the $S$ and the other letters also made the $S$ a very difficult target. In fact, the $S$ was the only target on which the number of location errors exceeded the number of identification errors. Every subject made more identity and location errors on the $S$ than on any other letter target. Although no clear pattern of errors was present in the identity responses to the $S$, a definite trend was found in the location errors. The location of the target $S$ was most likely to be reported as the location of the distractors 5 or 3 .

This pattern of location errors strongly implicates the role of physical information in the ability to locate, or "spatially tag," a BC target. Since the S was generally more physically similar to the digits than to the other letters, it was actually treated as a digit in the search. In the search for digits among letters, when the location of a target was reported incorrectly, the location of the $S$ was more likely to be reported than that of any other letter. In the search for letters among digits, the $S$ was the only target on which subjects made more location errors than identity errors. Thus, performance with the target $S$ was what would be expected to be seen in a WC search.

In summary, with (1) insufficient time to extract both types of information and (2) sufficient physical differences between the target and distractors, subjects were able to locate a BC target more accurately than they were able to identify it. For all of the targets except the $S$, the data suggest that knowledge of a BC target's location precedes its full identification. The data also suggest that physical information plays a crucial role in this process. A BC target must be sufficiently physically different from its opposite-category distractors for its location to be selected accurately and efficiently.

In a WC search, it should not be possible to "zero-in" on the target. Consequently, each item in the display should have to be examined in order to determine whether or not it is the target. Experiment 4 tested the assumption that a WC target must be fully identified before it can be located.

\section{EXPERIMENT 4}

\section{Method}

Design. The design of Experiment 4 was the same as that of Experiment 3 with two exceptions. First, in Experiment 4, only one task was performed (by one group of subjects), and that was a WC search task with letter targets appearing among letter distractors. Second, since the order of display durations used in Experiment 3 had no effect, and since the differences between the ID and LOC functions found in Experiment 3 were apparent only at the three lowest display durations, only these three display durations were used in Experiment 4. All other details were identical to those of Experiment 3.

Subjects. The subjects were 12 undergraduate students at Brown University. All reported normal or corrected-to-normal vision and were paid for their participation.

Apparatus. The apparatus used was the same as that described for Experiment 3.

Stimulus materials. The stimulus materials used were the same as those used in Experiment 3 with one exception. The digits from the digit-in-letter search in Experiment 3 were replaced by eight 
new letter targets in Experiment 4. The targets were A, E, O, U, $\mathrm{B}, \mathrm{P}, \mathrm{F}$, and $\mathrm{T}$ (averaging $.18^{\circ}$ in width), and the distractors were the C, D, G, H, K, R, S, and V used in Experiment 3. These characters are displayed in Figure 4.

Procedure. The procedure of Experiment 4 was the same as that used in Experiment 3.

\section{Results and Discussion}

The results of this experiment are displayed in Table 9. The differences between the ID and LOC functions indicate that mean accuracies were higher for ID responses than for $L O C$ responses at all three display durations. Although they were corrected for guessing and analyzed in the same fashion as the results in Experiment 3, both the corrected and the uncorrected data are presented in the table. Again, the corrected responses were analyzed for the effects of the order of display durations and order of ID and LOC responses. Neither of these effects and none of their interactions with the effect of ID versus LOC responses were significant $[F(1,8) \leq 3.4, p>.05]$. The effects were therefore excluded from further analysis.

A two-factor repeated-measures analysis of variance (identification vs. location responses $\times$ display duration) was performed on the data. Again, display duration had a significant effect, with accuracies increasing as the display duration increased $[F(2,22)=39.3, p<.01]$. As predicted, identification responses were significantly more accurate than location responses $[F(1,11)=43.7$, $p<.01$ ] [The same analysis on the uncorrected data showed the same effect; $F(1,11)=19.0, p<.01$.] The interaction of these two factors was not reliable $(F<1)$, since the effect was as strong with a display duration of $25 \mathrm{msec}$ as it was with a display duration of $10 \mathrm{msec}$.

The results of this experiment show that, under limited viewing conditions, it is easier to identify a target in a WC search than it is to report its precise location. This pattern of results is the opposite of that found in a BC search. A comparison of the results of the two experiments shows that the WC search was a much more difficult task than either BC search. The overall mean accuracy for the search for letters among letters was $28 \%$, $38 \%$ for the search for digits among letters (in the first three display durations) and $61 \%$ for the search for letters among digits (in the first three display durations).

Thus, it was found that a BC search was much more efficient than a WC search, and this difference in efficiency was due to the ability to locate the $\mathrm{BC}$ target for further processing. With the same distractor set, it was shown that in a BC search it is easier to locate a target than to identify it and that the opposite is true for a WC

\section{TARGETS: $\quad A E O U$ B P T}

\section{DISTRACTORS: C D G H K R S V}

Figure 4. Targets and distractors used in Experiment 4.
Table 9

Mean Response Accuracies for Location (LOC) and Identification (ID) Responses and the Differences Between Them as a Function of Display Duration for Experiment 4

\begin{tabular}{lccc}
\hline & \multicolumn{3}{c}{ Display Duration } \\
\cline { 2 - 4 } & 10 & 15 & 20 \\
\hline Corrected LOC & .13 & .23 & .37 \\
Corrected ID & .22 & .30 & .45 \\
LOC-ID & -.09 & -.07 & -.08 \\
Uncorrected LOC & .27 & .36 & .49 \\
Uncorrected ID & .32 & .39 & .52 \\
LOC-ID & -.05 & -.03 & -.03 \\
\hline
\end{tabular}

search-as long as the viewing conditions are limited and there are sufficient physical differences between the target and the distractors. With longer display durations, there was sufficient time to accurately identify and locate the BC target. This supports Jonides and Gleitman's idea of a "spatial tagging" of the categorically different item, with one important stipulation. The results of these experiments suggest that $\mathrm{BC}$ targets are not tagged because they are categorically different from the distractors, but because they are physically distinct. In Experiment 4, the targets $T$ and $F$ were as narrow as the $S$. Yet these items were not confused with the target $S$ as the 5 was in Experiment 3 . Thus, while uppercase letters are generally wider than digits, the specific physical features of these items are also crucial in the discrimination of one character from another.

Attempts to list the features that distinguish uppercase letters from digits may be futile, if not misguided. If a distinguishing set of features could be devised, it would surely change with different fonts and typefaces. Thus, any tenable set of features would have limited predictive value. Nevertheless, attempts have been made to devise a set of distinguishing features for capital letters (Geyer \& DeWald, 1973; Gibson, 1969). However, the present work and that of Townsend (1971) suggest that, in a consideration of the discriminability of letters and digits, a measure of overall similarity without regard to features may be more useful than a definition of the distinguishing features. Trying to parcel letters and digits into features is probably misguided because such familiar patterns are probably analyzed as whole patterns.

L. Allmendinger (personal communication, 1985) has described some general differences in the shapes of uppercase letters and digits. First, he notes that letters fill spaces of varying width, whereas digits (with the exception of the 1) fill spaces of constant width. This is a key feature of digits inasmuch as they are often used in columns. Second, digits have downstrokes that go from right to left, whereas letters that have such right-to-left downstrokes always also have left-to-right downstrokes. Third, curves in letters generally flatten on top and bottom (as in B, D, G, etc.); curves in digits do not (as in $2,3,5,6,8$, and 9). Allmendinger attributes these and other differences to the Arabic origin of digits and the Western origin of letters. It is important to note that 
although these differences are generally true for prototypical letters and digits, they will not hold for all typefaces.

Unlike Experiment 2, Experiment 4 does not allow for a differentiation between the effects of physical and category information. However, it does show that the typical features of the letters used in this experiment do not allow a target to be selected from its distractors as efficiently in a WC search as in the BC search of Experiment 3.

\section{GENERAL DISCUSSION}

The results of these experiments suggest that in a between-category search a preliminary analysis selects the target candidate on the basis of physical dissimilarity and zeros in on this item as the target before a complete analysis of features and identification take place. Therefore, different-category targets are selected from their distractors on the basis of their physical characteristics, not on the basis of category information per se. In this way, in a BC search, a character can be selected for further processing on the basis of an overall physical difference, for example, being wider than the other items in the display; it need not match a category or even a specific physical configuration.

In a BC search, the physical differences between the targets and distractors are usually sufficient to allow the target's location to be selected for further processing. Similarly, the features of items within a category are usually sufficiently similar to insure that a WC target must be identified before it can be located. This does not mean to imply that the WC target is identified without any knowledge of the target's location or configuration, but rather that it is not located as efficiently as the BC target. Thus, the ability to select the correct location for further processing is limited to a $\mathrm{BC}$ search because it is constrained by the physical features of the targets and distractors. An item that is physically very similar to a target may be localized as a target, whether or not it belongs to the same category as the target.

In summary, the data from this series of experiments suggest that the alphanumeric category effect is an artifact of the physical features of letters and digits. That is, the category effect can be explained by sensory effects that are directly related to the physical aspects of the stimulus and without reference to categories. Any facilitation found in one type of search (BC or WC) over another is most likely due to physical differences between the target and distractors that allow for quicker identification of the target. In this way, an alphanumeric search is no different from any other search task. The more physically different the target is from its distractors, the easier the search will be.

It must also be recognized that other factors, such as task demands and practice, can be as important as the physical characteristics of the targets and distractors in determining the potential for perceptual selectivity in visual search. Certain physical cues (e.g., shape or color) may be strong enough to demonstrate perceptual selectivity in some tasks but not in others (see Francolini \& Egeth, 1979). More importantly, practice with specific targets can lead to dramatic results. Hock, Rosenthal, and Stenquist (1985) have shown that practice can overcome the tendency of subjects to make false-alarm errors in a BC search with characters that belong to the same category as the target. Gleitman and Jonides (1976) were the first to demonstrate this catch-trial effect. They found that, after many trials on a BC search, the subjects responded to $\mathbf{a}$ as if it had been one of the targets 2 or 4 (i.e., by indicating "target present"). Hock et al. showed that with some practice (192 trials as opposed to 96) subjects responded correctly on the catch trial (i.e., reported "target absent'), but the response times for these responses were consistently longer than the response times for the other correct "target-absent" responses. With further practice (384 trials), this difference in response time was eliminated. Thus, with training on specific targets, subjects were able to adjust to the specific features and font of the targets and to fine-tune their search. The distractor 5 , physically and categorically similar to the target 3 , no longer proved to be distracting.

This is similar to the case in which, after extensive practice, subjects developed an automatic attention response to specific targets (Schneider \& Shiffrin, 1977). In this case, the targets were not inherently dissimilar from their distractors (e.g., as with the display of all letters), but with sufficient practice, perfect perceptual selectivity occurred and the targets were extracted from their backgrounds with little or no subjective effort. Although such practice results in search characteristics that are similar to those shown in demonstrations of the category effect, it is clear that they result from distinctly different processes. One process is the result of experience and is constrained by the amount of specific practice; the other is the result of an inherent ability to automatically select a physically distinct item from a visual array and is constrained by the physical similarities between targets and distractors. With automatic detection as the result of practice, it is not necessary for targets to be physically distinct from the distractors; extensive practice enables subjects to develop automatic attention responses to a few specific patterns. Demonstrations of the category effect, however, require the targets to be more physically similar to the items in their own category than to items in the opposite category so that the target can be easily, if not automatically, extracted from its background in the earliest stages of processing. No practice is necessary to demonstrate the effect, although extensive practice can help to refine the perceptual selectivity.

Interestingly, Schneider and Shiffrin (1977) explain the category effect as being due to an automatic attention response that is easily developed to category-level information. They explain the lack of a category effect noted in one study by suggesting that "an average physical difference" between a target and its distractors is used 
as an automatic detection cue that takes priority over ... category name" (p. 42). It is proposed here that the category effect can be demonstrated only when such an average physical difference between letters and digits exists in the display and can be used by the subjects as an automatic detection cue to direct attention to the target.

\section{REFERENCES}

BRAND, J. (1971). Classification without identification in visual search. Quarterly Journal of Experimental Psychology, 23, 178-186.

Butler, T. W. (1975). Luminance-duration relationships in the photopic ERG and the apparent brightness of flashes. Vision Research, 15, 693-698.

CoRCoRAN, D. W. J., JACKSON, A. (1977). Basic processes and strategies in visual search. In S. Dornic (Ed.), Attention and performance VI. Hillsdale, NJ: Erlbaum.

DunCan, J. (1983). Category effects in visual search: A failure to replicate the "oh-zero" phenomenon. Perception \& Psychophysics, 34, 221-232.

Egeth, H., Atkinson, J., Gilmore, G., \& Marcus, N. (1973). Factors affecting processing mode in visual search. Perception \& Psychophysics, 13, 394-402.

Francolini, C. M., \& Egeth, H. E. (1979). Perceptual selectivity is task dependent: The pop-out effect poops out. Perception \& Psychophysics, 25, 99-110.

GeYER, L. H., DEWALD, C. G. (1973). Feature lists and confusion matrices. Perception \& Psychophysics, 14, 471-482.

GiBson, E. J., (1969). Principles of perceptual learning and development. New York: Appleton-Century-Crofts.

GleitMan, H., Jonides, J. (1976). The cost of categorization in visual search: Incomplete processing of targets and field items. Perception \& Psychophysics, 20, 281-288.

Gleitman, H., \& Jonides, J. (1978). The effect of set on categorization in visual search. Perception \& Psychophysics, 24, 361-368.

Hock, H. S., Rosenthal, A., \& STENQuist, P. (1985). The category effect in visual search: Practice effects on catch trials. Perception \& Psychophysics, 37, 73-80.

INGLING, N. W. (1972). Categorization: A mechanism for rapid information processing. Journal of Experimental Psychology, 94, 239-243.

Jonides, J., \& Gleitman, H. (1972). A conceptual category effect in visual search: $O$ as a letter or a digit. Perception \& Psychophysics, $12,457-460$.

JoniDEs, J., \& Gleitman, H. (1976). The benefit of categorization in visual search: Target location without identification. Perception \& Psychophysics, 20, 289-298.

KRUEGER, L. E. (1984). The category effect in visual search depends on physical rather than conceptual differences. Perception \& Psychophysics, 35, 558-564.

Mollon, J. D., Polden, P. G. (1978). On the time constants of tachistoscopes. Quarterly Journal of Experimental Psychology, 30, 555-566.

NeIsSER, U. (1964). Visual search. Scientific American, 210, 94-102.

RABBIT, P. M. (1967). Learning to ignore irrelevant information. American Journal of Psychology, 80, 1-13.

SANTEe, J. L., \& Egeth, H. E. (1982). Do reaction time and accuracy measure the same aspects of letter recognition? Joumal of Experimental Psychology: Human Perception \& Performance, 8, 489-501.

SChNeIder, W., SHIFrrin, R. M. (1977). Controlled and automatic human information processing: I. Detection, search, and attention. Psychological Review, 84, 1-66.

Stelmach, L. B. (1984). Does rate of processing determine ease of target detection? Joumal of Experimental Psychology: Human Perception \& Performance, 10, 108-118.

TownSEND, J. T. (1971). Theoretical analysis of an alphabetic confusion matrix. Perception \& Psychophysics, 9, 40-49.

WiNER, B. J. (1971). Statistical principles in experimental design (2nd ed.). New York: McGraw-Hill.

\section{NOTES}

1. The display duration at which subjects reached criterion varied from 15 to 150 msec among subjects. Generally, the subjects who received the high-to-low order of display durations reached the criterion at a lower display duration than did the subjects who received the low-to-high order. The combined mean from Experiments 1 and 2 was $54 \mathrm{msec}(S D=48)$. The mean display duration at which criterion was reached for subjects who received the high-to-low order of display duration was $34 \mathrm{msec}$ $(S D=33)$; for the low-to-high group, the mean was $74 \mathrm{msec}(S D=53)$.

2. To ensure that the targets used were no more discriminable in one typeface than in the other, a preliminary experiment was run. Using the apparatus and procedure described above, 8 subjects identified individually presented targets first in one typeface and then in the other. Four subjects had the letter targets (I, E, S, and L), and 4 subjects had the digit targets $(1,3,5$, and 7$)$. The same five display durations used in Experiments 1 and 2 were also used, with the orders of display duration and typeface balanced across subjects. There were no differences between the mean accuracies for the L.C.D. and Futura Medium (F.M.) typefaces averaged across display duration (letters, L.C.D. $=79.9$, F.M. $=78.9$; digits, L.C.D. $=85.2$, F.M. $=84.7$ ) or even at the lowest display duration [letters, L.C.D. $=41.6(S D=12)$, F.M. $=38.5$ $(S D=5)$; digits, L.C.D. $=42.7(S D=21)$, F.M. $=45.8(S D=16)]$ Mean response times were similarly unaffected by typeface [letters, L.C.D. $=627 \mathrm{msec}(S D=196)$, F.M. $=620 \mathrm{msec}(S D=180)$; digits, L.C.D. $=525 \mathrm{msec}(S D=39)$, F.M. $=546 \mathrm{msec}(S D=57)]$. Although this control experiment does not address the matter of how the characters are perceived in multielement arrays, it does suggest that the targets in the two typefaces were equally discriminable.

3. The mean overall accuracy was $88 \%$ ( $90 \%$ for letter targets and $86 \%$ for digit targets) in Experiment 1 and $86 \%$ (85\% for letter targets and $87 \%$ for digit targets) in Experiment 2. Overall mean RT was $696 \mathrm{msec}$ ( 719 for letter targets and 674 for digit targets) in Experiment 1 and $695 \mathrm{msec}$ (706 for letter targets and 685 for digit targets) in Experiment 2. This suggests that the overall discriminability of the targets among their distractors was comparable in the two typefaces.

4. Recall that trials were blocked by display duration. After each block of trials, the subjects were asked to estimate the average number of distractors they could have identified and located accurately had they been asked to do so at the end of each trial. This information was used to assess the probability of reporting a correct target location by chance. For example, if a subject noticed that distractor $X$ was in the west position and distractor $Y$ was in the north position, then the chances of reporting the target's correct location by chance became 1 in 6 rather than 1 in 8. Meanwhile, the probability of reporting the target's correct identity by chance remained 1 in 8 . Because of the possibility that this discrepancy might have inflated location accuracies, all response accuracies were corrected for guessing by using the following formula:

$$
P(\text { obtained })=P(\text { true })+1 / n[1-P(\text { true })],
$$

where: $P$ (obtained) is the observed probability of a correct response; $P$ (true) is the probability of an accurate response corrected for guessing; $n=8$ for identification responses; and $n=8$ minus the number of distractors seen, for location responses.

5. Any effects found here due to relatively low display durations may also be attributable to the accompanying low brightness levels since apparent brightness and duration are known to covary when low display durations are used (see Butler, 1975). However, in a study in which these two factors were varied independently, they were found to each have the same effect on a target-detection task (Stelmach, 1984). It should also be noted that, due to the constraints of the tachistoscope, the display durations and luminance levels cited in the present experiments are not exact for durations below $50 \mathrm{msec}$ (see Molion \& Polden, 1978).

6. With digits as targets, an average of $25 \%$ of all errors involved either identifying the target as a 5 when a 5 was not present or correctly identifying the target as a 5 but reporting the location incorrectly. In $60 \%$ of these errors, the item was identified as a 5 and its location was reported as that occupied by the distractor $S$. That is, these items were identified (either correctly or incorrectly) as a $\mathbf{5}$ and said to be located 
where the $\mathrm{S}$ was. Analysis of the responses with the target correctly identified as a 5 but incorrectly located revealed that $80 \%(S D=17 \%)$ of these errors could be attributed to localizing the 5 as the $S$. Analysis of all location errors showed that $30 \%$ of them were attributable to localizing the target where the $S$ was positioned. This is almost three times the $10.9 \%$ expected with errors randomly distributed. When the targets were correctly identified, yet located in the position occupied by the distractor S, most (77\%) of those targets were 5s. Yet such errors were not limited to targets that were physically very similar to the S and included the targets $1,3,4$, and 7 . Clearly, for subjects searching for "any digit," the $\mathrm{S}$ was seen as a digit and localized as such.

(Manuscript received April 10, 1986; revision accepted for publication August 29, 1986.)

\section{ERRATUM}

Collyer, C. E. Comparing strong and weak models by fitting them to computer-generated data. Perception \& Psychophysics, 1985, 38, 476-481-The equation that appears on page 479 contains an error: The $\pi$ that should have been included is missing. The correct equation is:

$$
Y=A+B\left[\sin \left(2 \pi R_{1}\right)\right]\left(-2 \log _{e} R_{2}\right)^{1 / 2} .
$$

